\title{
6. VARIABLES AND CONSTANTS IN THE CURRICULUM FOR THE MUSIC SPECIALISATIONS OF THE ROMANIAN UNIVERSITY EDUCATION
}

Eugenia Maria Paşca ${ }^{295}$

\begin{abstract}
The Romanian academic system must adapt to the changing demands imposed by the European educational standards, according to the conventions of Bologna and Lisbon. The compatibilisation of university paths in creating the curricula must consider the competences which are to be acquired. The responsibility belongs equally to the institutions providing academic programs, but also to the Romanian Agency for Quality Assurance in Higher Education. We intend to analyse comparatively both the constant and variable elements of the academic programs for the Music field. The efficiency of these programs is particularly reflected from the point of view of insertion in the labour market, so the direct beneficiaries are the graduate students.
\end{abstract}

Key words: standards, curriculum, music specialisations, labour market

\section{Introduction}

We intend to make an analysis on the options of university institutions providing and ensuring Bachelor's degree programs in Romania, for the specialisations Musical pedagogy (6, named from U1 to U6) and Musical performance / Instruments and Canto (4, named from U1 to U4). We will monitor if they are compliant with the Romanian and European occupational standards, according to the competences proposed to be acquired by covering them. These educational programs are periodically reviewed, every 5 years, in order to comply with the dynamics of university and professional qualifications, according to the university autonomy, to the demands of Lisbon and Bologna Convention and to the demands of the labour market. Within the faculties' departments, periodical analyses of the academic programs are taking place, being attended by professors, students, graduates and employers' representatives, who identify the reviewing needs of the curricula/ syllabi, after which they are reviewed and regulated by the educational policies in force and provided in the regulations concerning the initiation, the approval, the monitoring and the periodical assessment of academic programs, existing in every higher education institution of Romania. At the same time, as a complementary procedure, the professors taking part in the mobilities must make reports regarding the academic programs of the European universities visited, proposing different improvements of the curriculum, by comparison, in order to perform the compatibility of these programs with the European ones.

\section{Method}

The comparative analysis of the academic programs proposed to be analysed in this study, valid for the academic year 2015-2016, will offer us an overview on the preparation of future specialists in performing or pedagogy-musical arts,

\footnotetext{
${ }^{295}$ Profesor PhD., "George Enescu" University of Arts from Iaşi of Romania, email:
} eugenia_maria_pasca@yahoo.com 
as graduates of the Bachelor's degree programs whose professional and transversal competences must correspond to the qualifications obtained. By means of periodical assessment procedures, reports and regulations are made, which permanently determine the change of curricula and syllabi according to the standards of the Romanian Agency for Quality Assurance in Higher Education - ARACIS / ARTS (1), as well as the opportunity of initiating, in the future, new programs, in line with the labour market dynamics. The first stage is represented by the internal assessment, and the second one is represented by the external one of the academic programs and considers several indicators which mainly reflect the correspondence between them and the qualifications which are to be obtained by graduates, such as the correspondence between the latter ones and the occupational field according to the Romanian National Qualifications Register for Higher Education - RNCIS (2, 3). The hypothesis consists in the fact that there should be a flexible constant of the disciplines' categories, as a name and division between fundamental, speciality or compulsory complementary disciplines, according to the amount of hours and credits allocated. The variables should exist between the difference between optional and facultative complementary disciplines, naturally determined by the didactic human resource available and the students' options.

\section{Discussions}

We will further analyse the Musical pedagogy program, with the pedagogic disciplines included in the curriculum, since the academic year 2015-2016 (4) in line with the professional qualifications (according to ISCO 08:2332 Secondary education teachers - 31) established by the Specific standards for the academic study programs of the Arts-Music Bachelor's Degree fields (1, pp. 49) and the Specific standards for the academic study programs of the Administrative Sciences, Education Sciences, Psychology Bachelor's Degree fields and Programs whose name and mission intend explicitly the professors' preparation for secondary education (6, p. 40). The relationship between disciplines and professional competences is direct and we notice that the first one is identical for all the programs proposed for analysis - $\mathrm{C} 1$. The performance of a correct correlation between the musical text and the audio configuration (chamber/symphonic, with a high complexity level) according to the musical listening and writing-reading skills acquired (including the inner musical hearing), as well as the 3 transversal competences:

TC1 Designing, organising, performing, coordinating and assessing an artistic event/learning unit, including specific contents of the musical disciplines' syllabus;

TC2 Applying the efficient labour techniques in a multidisciplinary team on various hierarchical levels;

TC3 Objective self-assessment of the professional formation need for the purpose of insertion and adaptability to the labour market demands.

The program's specificity is well differentiated and particularised by the following professional competences: 
C2 Correct use of classical and modern writing techniques and of fundamental methods of musical analysis;

C3 Analysis of musical works, on the basis of identification and description of their stylistic features and considering the cultural context;

$\mathrm{C} 4$ Correct and functional use of main elements of the performing technique (member and conductor of a choir, playing the piano);

C5 Elaboration of musicological and musical criticism works, by covering all the stages (from creation to accomplishment), by integrating the specific musical knowledge and skills acquired and by bringing the personal creativity and the analysis and synthesis abilities;

C6 Designing programs for the compulsory pre-university education students;

C7 Performing the activities related to the formation-education process of the compulsory pre-university education;

C8 Assessing the learning processes and the results recorded.

If until the academic year 2014-2015, the completion for didactic qualification was provided by compulsorily covering the additional courses module of the Teaching Staff Training Departments, from the academic year 2015-2016, the specific disciplines were included in this program $(6, p .40)$. This aspect is still in the debate of competent forums, because out of the total of 180 ECTS, the strictly musical field has 150 ECTS, the other 30 ECTS being represented by the psycho-pedagogical and didactic disciplines.

Table no. 1

\begin{tabular}{|c|c|c|c|c|c|c|c|c|}
\hline Ref. no. & Disciplines / categories of disciplines & U1 & $\mathbf{U 2}$ & U3 & U4 & U5 & U6 & Differences \\
\hline A. & $\begin{array}{l}\text { Fundamental compulsory disciplines } \\
30-35 \%\end{array}$ & & & & & & & \\
\hline 1. & Theory-music theory - dictation & $\mathrm{x}$ & $\mathrm{x}$ & $\mathrm{x}$ & $\mathrm{x}$ & $\mathrm{x}$ & $\mathrm{x}$ & \\
\hline 2. & History of music & $\mathrm{x}$ & $\mathrm{x}$ & $\mathrm{x}$ & $\mathrm{x}$ & $\mathrm{x}$ & $\mathrm{x}$ & \\
\hline 3. & Harmony & $\mathrm{x}$ & $\mathrm{x}$ & $\mathrm{x}$ & $\mathrm{x}$ & $\mathrm{x}$ & $\mathrm{x}$ & \\
\hline 4. & Musical forms and analyses & $\mathrm{x}$ & $\mathrm{x}$ & $\mathrm{x}$ & $\mathrm{x}$ & $\mathrm{x}$ & $\mathrm{x}$ & \\
\hline 5. & Polyphony - counterpoint and fugue & $\mathrm{x}$ & $\mathrm{x}$ & $\mathrm{x}$ & $\mathrm{x}$ & $\mathrm{x}$ & $\mathrm{x}$ & \\
\hline 6. & Educational psychology & $\mathrm{x}$ & $\mathrm{x}$ & $\mathrm{x}$ & $\mathrm{x}$ & $\mathrm{x}$ & $\mathrm{x}$ & \\
\hline B. & $\begin{array}{l}\text { Speciality compulsory disciplines } 50 \text { - } \\
55 \%\end{array}$ & & & & & & & \\
\hline 1. & Choir conducting & $\mathrm{x}$ & $\mathrm{x}$ & $\mathrm{x}$ & $\mathrm{x}$ & $\mathrm{x}$ & $\mathrm{x}$ & \\
\hline 2. & Musical education systems & $\mathrm{x}$ & $\mathrm{x}$ & $\mathrm{x}$ & $\mathrm{x}$ & $\mathrm{x}$ & $\mathrm{x}$ & \\
\hline 3. & $\begin{array}{l}\text { Theory of instruments and } \\
\text { instrumentation }\end{array}$ & $\mathrm{x}$ & $\mathrm{x}$ & $\mathrm{x}$ & $\mathrm{x}$ & $\mathrm{x}$ & $\mathrm{x}$ & \\
\hline 4. & Score reading & $\mathrm{x}$ & $\mathrm{x}$ & $\mathrm{x}$ & $\mathrm{x}$ & $\mathrm{x}$ & $\mathrm{x}$ & \\
\hline 5. & Choir canto & $\mathrm{x}$ & $\mathrm{x}$ & $\mathrm{x}$ & $\mathrm{x}$ & $\mathrm{x}$ & $\mathrm{x}$ & \\
\hline 6. & Musical hearing education systems & $\mathrm{x}$ & $\mathrm{x}$ & $\mathrm{x}$ & & & & 3 \\
\hline 7. & $\begin{array}{l}\text { Artistic } \\
\text { ensemble }\end{array}$ practice: choir/orchestra & $\mathrm{x}$ & $\mathrm{x}$ & $\mathrm{x}$ & $\mathrm{x}$ & $\mathrm{x}$ & $\mathrm{x}$ & \\
\hline 8. & $\begin{array}{l}\text { Musical education } \\
\text { vocal/instrumental groups }\end{array}$ & & $\mathrm{x}$ & & & & & 1 \\
\hline 9. & $\begin{array}{l}\text { Pedagogy I (Pedagogy foundations, } \\
\text { Theory and methodology of } \\
\text { curriculum) }\end{array}$ & $\mathrm{x}$ & $\mathrm{x}$ & $\mathrm{x}$ & $\mathrm{x}$ & $\mathrm{x}$ & $\mathrm{x}$ & \\
\hline 10. & $\begin{array}{l}\text { Pedagogy II (Theory and methodology } \\
\text { of education, Theory and practice of } \\
\text { assessment) }\end{array}$ & $\mathrm{x}$ & $\mathrm{x}$ & $\mathrm{x}$ & $\mathrm{x}$ & $\mathrm{x}$ & $\mathrm{X}$ & \\
\hline 11. & Speciality didactics & $\mathrm{x}$ & $\mathrm{x}$ & $\mathrm{x}$ & $\mathrm{x}$ & $\mathrm{x}$ & $\mathrm{x}$ & \\
\hline 12. & Pedagogic practice & $\mathrm{x}$ & $\mathrm{x}$ & $\mathrm{x}$ & $\mathrm{x}$ & $\mathrm{x}$ & $\mathrm{x}$ & \\
\hline
\end{tabular}




\begin{tabular}{|c|c|c|c|c|c|c|c|c|}
\hline 13. & $\begin{array}{l}\text { Technique of scientific research and } \\
\text { composition }\end{array}$ & $\mathrm{x}$ & $\mathrm{x}$ & $\mathrm{x}$ & $\mathrm{x}$ & $\mathrm{x}$ & $\mathrm{x}$ & \\
\hline 14. & Artistic practice/ Choir ensemble & $\mathrm{X}$ & $\mathrm{X}$ & $\mathrm{X}$ & $\mathrm{x}$ & $\mathrm{X}$ & $\mathrm{x}$ & \\
\hline 15. & Artistic practice/Orchestral ensemble & & $\mathrm{x}$ & $\mathrm{x}$ & & & $\mathrm{x}$ & 3 \\
\hline 16. & Musical liturgical practice & & & & & $\mathrm{x}$ & & 1 \\
\hline 17. & Protestant liturgics and hymnology & & & & & $\mathrm{x}$ & & 1 \\
\hline C. & $\begin{array}{ll}\text { Compulsory } & \text { complementary } \\
\text { disciplines } 10 \% & \\
\end{array}$ & & & & & & & \\
\hline 1. & Composers and musical literature & & $\mathrm{x}$ & & $\mathrm{x}$ & & & 2 \\
\hline 2. & History of pop culture & & $\mathrm{x}$ & & & & & 1 \\
\hline 3. & Piano & $\mathrm{x}$ & $\mathrm{X}$ & $\mathrm{X}$ & $\mathrm{x}$ & $\mathrm{x}$ & $\mathrm{x}$ & \\
\hline 4. & Physical education & $\mathrm{x}$ & $\mathrm{x}$ & $\mathrm{x}$ & $\mathrm{x}$ & $\mathrm{x}$ & $\mathrm{x}$ & \\
\hline 5. & Foreign language & $\mathrm{X}$ & $\mathrm{x}$ & $\mathrm{X}$ & $\mathrm{x}$ & $\mathrm{x}$ & $\mathrm{x}$ & \\
\hline 6. & $\begin{array}{l}\text { Musical information on computer / } \\
\text { Musical informatics }\end{array}$ & $\mathrm{x}$ & $\mathrm{x}$ & $\mathrm{x}$ & $\mathrm{x}$ & $\mathrm{x}$ & $\mathrm{x}$ & \\
\hline 7. & Musical folklore & $\mathrm{x}$ & $\mathrm{x}$ & $\mathrm{x}$ & $\mathrm{x}$ & $\mathrm{x}$ & $\mathrm{x}$ & \\
\hline 8. & Management of the class & $\mathrm{x}$ & $\mathrm{x}$ & $\mathrm{x}$ & $\mathrm{x}$ & $\mathrm{x}$ & $\mathrm{x}$ & \\
\hline 9. & Computer assisted training & $\mathrm{x}$ & $\mathrm{x}$ & $\mathrm{x}$ & $\mathrm{x}$ & $\mathrm{x}$ & $\mathrm{x}$ & \\
\hline 10. & History of Christian religion & & $\mathrm{x}$ & & & & & 1 \\
\hline D. & $\begin{array}{l}\text { Optional complementary disciplines } \\
10 \%\end{array}$ & & & & & & & \\
\hline 1. & $\begin{array}{l}\text { Religious music / Byzantine music / } \\
\text { Psaltic music }\end{array}$ & $\mathrm{x}$ & $\mathrm{x}$ & & $\mathrm{X}$ & & & 3 \\
\hline 2. & Musical historiography & & & & $\mathrm{x}$ & & & 1 \\
\hline 3. & Jazz module & $\mathrm{x}$ & & & $\mathrm{x}$ & & & 2 \\
\hline 4. & Protestant liturgics and hymnology & & & & & $\mathrm{x}$ & & 1 \\
\hline 5. & Popular instrument & & & $\mathrm{x}$ & & & & 1 \\
\hline 6. & Popular chant & & & $\mathrm{x}$ & & & & 1 \\
\hline 7. & Musical informatics & & & $\mathrm{x}$ & & & & 1 \\
\hline 8. & Sound musical production & & & $\mathrm{x}$ & & & & 1 \\
\hline 9. & Jazz/Jazz aesthetics & & & $\mathrm{x}$ & & & & 1 \\
\hline 10. & Musical management & $\mathrm{x}$ & $\mathrm{x}$ & & & & & 2 \\
\hline 11. & History of jazz & $\mathrm{x}$ & & & & & & 1 \\
\hline & Jazz module & & & & & & & \\
\hline 12. & Jazz music related groups & $\mathrm{x}$ & & & $\mathrm{x}$ & & & 2 \\
\hline 13. & Jazz improvisation & & & & $\mathrm{x}$ & & & 1 \\
\hline 14. & Jazz instrument & & & & $\mathrm{x}$ & & & 1 \\
\hline 15. & Spiritual counselling & & & & & $\mathrm{x}$ & & 1 \\
\hline 16. & Apologetics & & & & & $\mathrm{x}$ & & 1 \\
\hline 17. & Elements of musical composition & & & & & $\mathrm{x}$ & & 1 \\
\hline 18. & Introduction to musicotherapy & & & & & $\mathrm{x}$ & & 1 \\
\hline 19. & History of Baptists & & & & & $\mathrm{x}$ & & 1 \\
\hline 20. & Missiology & & & & & & & \\
\hline E. & Facultative disciplines $10 \%$ & & & & & & & \\
\hline 1. & Instrument & $\mathrm{X}$ & $\mathrm{x}$ & & $\mathrm{x}$ & $\mathrm{x}$ & $\mathrm{x}$ & 5 \\
\hline 2. & Canto & $\mathrm{X}$ & $\mathrm{x}$ & & $\mathrm{x}$ & $\mathrm{x}$ & $\mathrm{x}$ & 5 \\
\hline 3. & Acoustics and studio techniques & & & & & $\mathrm{x}$ & & 1 \\
\hline 4. & Instrument/chant didactics & & & & & $\mathrm{x}$ & & 1 \\
\hline 5. & Instrument/Chant teaching practice & & & & & $\mathrm{x}$ & & 1 \\
\hline 6. & Co-rehearsal & & & & & $\mathrm{x}$ & & 1 \\
\hline 7. & $\begin{array}{l}\text { Science of information and } \\
\text { communication }\end{array}$ & & & & & $\mathrm{x}$ & & 1 \\
\hline 8. & Philosophy of religion and culture & & & & & $\mathrm{x}$ & & 1 \\
\hline 9. & $\begin{array}{l}\text { Folkloric instrumental arrangements/ } \\
\text { popular music }\end{array}$ & $\mathrm{x}$ & & & & & & 1 \\
\hline \multirow[t]{2}{*}{10.} & $\begin{array}{lll}\text { Folkloric ensemble/Popular music } \\
\text { ensemble }\end{array}$ & $\mathrm{x}$ & & & & & & 1 \\
\hline & Traditional vocal music module & & & & & & & \\
\hline 11. & Folkloric chant & & & $\mathrm{x}$ & & $\mathrm{x}$ & $\mathrm{x}$ & 3 \\
\hline 12. & Traditional / folkloric ensemble & & & $\mathrm{x}$ & & $\mathrm{x}$ & $\mathrm{x}$ & 3 \\
\hline
\end{tabular}




\begin{tabular}{|c|l|c|c|c|c|c|c|c|}
\hline 13. & $\begin{array}{l}\text { Grading and restoring traditional vocal } \\
\text { music }\end{array}$ & & $\mathrm{x}$ & & $\mathrm{x}$ & & 2 \\
\hline & $\begin{array}{l}\text { Performing module of popular/jazz } \\
\text { music }\end{array}$ & & & & & & \\
\hline 14. & Performing of popular/jazz music & & & $\mathrm{x}$ & & $\mathrm{x}$ & & 2 \\
\hline 15. & Estrada/pop/rock ensemble & & & $\mathrm{x}$ & & $\mathrm{x}$ & & 2 \\
\hline 16. & Instrumental and vocal arrangement & & $\mathrm{x}$ & & $\mathrm{x}$ & & 2 \\
\hline
\end{tabular}

The Musical performance program, in correspondence with the professional qualifications (according to ISCO 08:2652 - Musicians, singers and composers - 31) established by the Specific standards for Bachelor's degree academic education programs in the field Arts - Music (1, p. 4-9). The first specialisation is the one regarding the Instrumental performance (5) and where we identify correlations between professional competences and Canto competences (5):

C2 Identifying and describing the characteristics of different cultures, stylistic periods and musical genres, as well as the correct use of main elements of classical writing and musical analysis techniques;

C3 Analysing and performing a representative selection of the soloist repertoire intended for the instrument studied, using the necessary instrumental technical and performing skills and integrating the musical knowledge and skills acquired; C5 Expressing the own performing style, starting from choosing, approaching, constructing and studying the repertoire until the public presentation of the instrumental/vocal performance creation.

A particularity is represented by the $\mathrm{C} 4$ competence - Analysing and performing a representative selection of the chamber/symphony repertoire including the instrument studied, using both auditory and instrumental technical and performance skills required for the integration into an ensemble (chamber groups, orchestra ensembles) and the musical specific knowledge and skills acquired.

Table no. 2

\begin{tabular}{|c|c|c|c|c|c|c|}
\hline Ref. no. & Disciplines / categories of disciplines & U1 & $\mathbf{U 2}$ & U3 & $\mathbf{U} 4$ & Differences \\
\hline A. & Fundamental compulsory disciplines $\mathbf{3 0 - 3 5 \%}$ & & & & & \\
\hline 1. & Theory-Music theory-dictation & $\mathrm{x}$ & $\mathrm{x}$ & $\mathrm{x}$ & $\mathrm{x}$ & \\
\hline 2. & History of music & $\mathrm{x}$ & $\mathrm{x}$ & $\mathrm{x}$ & $\mathrm{x}$ & \\
\hline 3. & Harmony & $\mathrm{x}$ & $\mathrm{x}$ & $\mathrm{x}$ & $\mathrm{x}$ & \\
\hline 4. & Musical forms and analyses & $\mathrm{x}$ & $\mathrm{x}$ & $\mathrm{x}$ & $\mathrm{x}$ & \\
\hline 5. & Polyphony - counterpoint and fugue & $\mathrm{x}$ & $\mathrm{x}$ & $\mathrm{x}$ & $\mathrm{x}$ & \\
\hline B. & Speciality compulsory disciplines $50-55 \%$ & & & & & \\
\hline 1. & Instrument & $\mathrm{x}$ & $\mathrm{x}$ & $\mathrm{x}$ & $\mathrm{x}$ & \\
\hline 2. & Chamber music & $\mathrm{x}$ & $\mathrm{x}$ & $\mathrm{x}$ & $\mathrm{x}$ & \\
\hline 3. & Accompaniment & $\mathrm{x}$ & $\mathrm{x}$ & $\mathrm{x}$ & $\mathrm{x}$ & \\
\hline 4. & Study of accompaniment & $\mathrm{x}$ & $\mathrm{x}$ & $\mathrm{x}$ & $\mathrm{x}$ & \\
\hline 5. & Orchestra & $\mathrm{x}$ & $\mathrm{x}$ & $\mathrm{x}$ & $\mathrm{x}$ & \\
\hline 6. & Artistic practice & $\mathrm{x}$ & $\mathrm{x}$ & $\mathrm{x}$ & $\mathrm{x}$ & \\
\hline 7. & Artistic management & $\mathrm{x}$ & $\mathrm{x}$ & $\mathrm{x}$ & $\mathrm{x}$ & \\
\hline 8. & Choral ensemble & & & & $\mathrm{x}$ & 1 \\
\hline 9. & Repertoire analyses & & $\mathrm{x}$ & $\mathrm{x}$ & & 2 \\
\hline 10. & Instrument tuning & & & $\mathrm{x}$ & & 1 \\
\hline 11. & Accompaniment figured bass & & & $\mathrm{x}$ & $\mathrm{x}$ & 2 \\
\hline 12. & Improvisation & $\mathrm{x}$ & & & & 1 \\
\hline
\end{tabular}




\begin{tabular}{|c|c|c|c|c|c|c|}
\hline 13. & Performance theory elements & $\mathrm{x}$ & $\mathrm{x}$ & & & 2 \\
\hline C. & Compulsory complementary disciplines $10 \%$ & & & & & \\
\hline 1. & Foreign language & $\mathrm{x}$ & $\mathrm{x}$ & $\mathrm{x}$ & $\mathrm{x}$ & \\
\hline 2. & Physical education & $\mathrm{x}$ & $\mathrm{x}$ & $\mathrm{x}$ & $\mathrm{x}$ & \\
\hline 3. & Compared performances & & $\mathrm{x}$ & & & 1 \\
\hline 4. & Musical information on computer & $\mathrm{x}$ & $\mathrm{x}$ & $\mathrm{x}$ & $\mathrm{x}$ & \\
\hline 5. & Musical aesthetics & $\mathrm{x}$ & $\mathrm{x}$ & $\mathrm{x}$ & $\mathrm{x}$ & \\
\hline 6. & Musical folklore & $\mathrm{x}$ & $\mathrm{x}$ & $\mathrm{x}$ & $\mathrm{x}$ & \\
\hline 7. & Musical culture and practice & & $\mathrm{x}$ & & $\mathrm{x}$ & 2 \\
\hline D. & Optional complementary disciplines $10 \%$ & & & & & \\
\hline 1. & Orchestra studies & & & & $\mathrm{x}$ & 1 \\
\hline 2. & Complementary instrument & & $\mathrm{x}$ & & $\mathrm{x}$ & 2 \\
\hline 3. & Sound production & & $\mathrm{x}$ & $\mathrm{x}$ & & 2 \\
\hline 4. & Jazz & & & $\mathrm{x}$ & & 1 \\
\hline 5. & Vocal arrangement & & & $\mathrm{x}$ & & 1 \\
\hline 6. & Baroque music & $\mathrm{x}$ & & & & 1 \\
\hline 7. & Choir accompaniment & $\mathrm{x}$ & & & & 1 \\
\hline 8. & Contemporaneous music & $\mathrm{x}$ & & & & 1 \\
\hline 9. & Score reading & $\mathrm{x}$ & & & & 1 \\
\hline \multirow[t]{2}{*}{10.} & History of pianistic art & $\mathrm{x}$ & & & & 1 \\
\hline & Jazz module & & & & & \\
\hline 11. & Jazz music specific groups & $\mathrm{x}$ & & & $\mathrm{x}$ & 2 \\
\hline 12. & Jazz improvisation & & & & $\mathrm{x}$ & 1 \\
\hline 13. & Jazz instrument & & & & $\mathrm{x}$ & 1 \\
\hline E. & Facultative disciplines $10 \%$ & & & & & \\
\hline 1. & European culture and civilisation & $\mathrm{x}$ & & & & 1 \\
\hline 2. & Optional instrument & & & $\mathrm{x}$ & $\mathrm{x}$ & 2 \\
\hline 3. & Canto & & & $\mathrm{x}$ & $\mathrm{x}$ & 2 \\
\hline 4. & Popular music/Jazz performance & & & $\mathrm{x}$ & & 1 \\
\hline 5. & Orchestra conducting & & & $\mathrm{x}$ & & 1 \\
\hline 6. & Alternative performance currents & & & $\mathrm{x}$ & & 1 \\
\hline 7. & Informatics & $\mathrm{x}$ & & & & 1 \\
\hline \multirow[t]{2}{*}{8.} & Musical semiotics & $\mathrm{x}$ & & & & 1 \\
\hline & Traditional vocal music module & & & & & \\
\hline 9. & Folkloric chant & & & $\mathrm{x}$ & $\mathrm{x}$ & 2 \\
\hline 10. & Traditional / folkloric ensemble & $\mathrm{x}$ & & $\mathrm{x}$ & $\mathrm{x}$ & 3 \\
\hline \multirow[t]{2}{*}{11.} & Grading and restoring traditional vocal music & & & $\mathrm{x}$ & & 1 \\
\hline & Performing module of popular/jazz music & & & & & \\
\hline 12. & Performing of popular/jazz music & & & $\mathrm{x}$ & & 1 \\
\hline 13. & Estrada/pop/rock ensemble & $\mathrm{x}$ & & $\mathrm{x}$ & & 2 \\
\hline \multirow[t]{2}{*}{14.} & Instrumental and vocal arrangement & & & $\mathrm{x}$ & & 1 \\
\hline & Liturgical repertoire module & & & & & \\
\hline 15. & Historic instrument & & & $\mathrm{x}$ & & 1 \\
\hline 16. & Liturgical accompaniment & & & $\mathrm{x}$ & & 1 \\
\hline 17. & Choir conducting & & & $\mathrm{x}$ & & 1 \\
\hline
\end{tabular}

For the Musical performance program, in correspondence with the professional qualifications (according to ISCO 08:2652 - Musicians, singers and composers - 31) established by the Specific standards for Bachelor's degree academic education programs in the field Arts - Music (1, p. 4-9). For the second specialisation, which refers to Canto (5), the competence $\mathrm{C} 4$ Using hearing, vocal, linguistic, kinaesthetic and expressive skills required for the musical and theatrical representation regarding the lyric performance, intending the integration in the musical ensemble (musical theatre), the integration in the work dramaturgy, networking with the other characters, stage movement and other aspects of stage performance, is the one particularising this program. 
Table no. 3

\begin{tabular}{|c|c|c|c|c|c|c|}
\hline Ref. no. & Disciplines / categories of disciplines & U1 & U2 & U3 & $\mathrm{U} 4$ & Differences \\
\hline A. & Fundamental compulsory disciplines $30-35 \%$ & & & & & \\
\hline 1. & Theory-music theory - dictation & $\mathrm{x}$ & $\mathrm{x}$ & $\mathrm{x}$ & $\mathrm{x}$ & \\
\hline 2. & History of music & $\mathrm{x}$ & $\mathrm{x}$ & $\mathrm{x}$ & $\mathrm{x}$ & \\
\hline 3. & Harmony & $\mathrm{x}$ & $\mathrm{x}$ & $\mathrm{x}$ & $\mathrm{x}$ & \\
\hline 4. & Musical forms and analyses & $\mathrm{x}$ & $\mathrm{x}$ & $\mathrm{x}$ & $\mathrm{x}$ & \\
\hline 5. & Polyphony - counterpoint and fugue & $\mathrm{x}$ & $\mathrm{x}$ & $\mathrm{x}$ & $\mathrm{x}$ & \\
\hline B. & Speciality compulsory disciplines $50-55 \%$ & & & & & \\
\hline 1. & Canto & $\mathrm{x}$ & $\mathrm{x}$ & $\mathrm{x}$ & $\mathrm{x}$ & \\
\hline 2. & Canto accompaniment & $\mathrm{x}$ & $\mathrm{x}$ & $\mathrm{x}$ & $\mathrm{x}$ & \\
\hline 3. & Opera / Opera class & $\mathrm{x}$ & $\mathrm{x}$ & $\mathrm{x}$ & $\mathrm{x}$ & \\
\hline 4. & Opera class accompaniment & $\mathrm{x}$ & $\mathrm{x}$ & $\mathrm{x}$ & $\mathrm{x}$ & \\
\hline 5. & Lied-oratorio & $\mathrm{x}$ & $\mathrm{x}$ & $\mathrm{x}$ & $\mathrm{x}$ & \\
\hline 6. & Lied/oratorio accompaniment & $\mathrm{x}$ & $\mathrm{x}$ & $\mathrm{x}$ & $\mathrm{x}$ & \\
\hline 7. & Make-up & $\mathrm{x}$ & $\mathrm{x}$ & $\mathrm{x}$ & $\mathrm{x}$ & \\
\hline 8. & Art of the actor & $\mathrm{x}$ & $\mathrm{x}$ & $\mathrm{x}$ & $\mathrm{x}$ & \\
\hline 9. & Stage movement & $\mathrm{x}$ & $\mathrm{x}$ & $\mathrm{x}$ & $\mathrm{x}$ & \\
\hline 10. & Speaking & $\mathrm{x}$ & $\mathrm{x}$ & $\mathrm{x}$ & $\mathrm{x}$ & \\
\hline 11. & Artistic practice & $\mathrm{x}$ & $\mathrm{x}$ & $\mathrm{x}$ & $\mathrm{x}$ & \\
\hline C. & Compulsory complementary disciplines $10 \%$ & & & & & \\
\hline 1. & Foreign language & $\mathrm{x}$ & $\mathrm{x}$ & $\mathrm{x}$ & $\mathrm{x}$ & \\
\hline 2. & General piano & $\mathrm{x}$ & $\mathrm{x}$ & $\mathrm{x}$ & $\mathrm{x}$ & \\
\hline 3. & Physical education & $\mathrm{x}$ & $\mathrm{x}$ & $\mathrm{x}$ & $\mathrm{x}$ & \\
\hline 4. & Musical information on computer & $\mathrm{x}$ & $\mathrm{x}$ & $\mathrm{x}$ & $\mathrm{x}$ & \\
\hline 5. & Artistic/cultural management & $\mathrm{x}$ & $\mathrm{x}$ & $\mathrm{x}$ & $\mathrm{x}$ & \\
\hline 6. & History of theatre costumes & & & $\mathrm{x}$ & & 1 \\
\hline 7. & Vocal hygiene & & $\mathrm{x}$ & $\mathrm{x}$ & $\mathrm{x}$ & 3 \\
\hline 8. & $\begin{array}{l}\text { Scientific research and writing technique / } \\
\text { Preparation of the Bachelor's thesis }\end{array}$ & $\mathrm{x}$ & $\mathrm{x}$ & $\mathrm{x}$ & $\mathrm{x}$ & \\
\hline 9. & Sound musical production & & $\mathrm{x}$ & $\mathrm{x}$ & & 2 \\
\hline 10. & Musical aesthetics & $\mathrm{x}$ & $\mathrm{x}$ & $\mathrm{x}$ & $\mathrm{x}$ & \\
\hline 11. & Musical folklore & $\mathrm{x}$ & $\mathrm{x}$ & $\mathrm{x}$ & $\mathrm{x}$ & \\
\hline 12. & Musical culture & & $\mathrm{x}$ & & $\mathrm{x}$ & 2 \\
\hline 13. & Theory of musical performance & $\mathrm{x}$ & $\mathrm{x}$ & & & 2 \\
\hline D. & Optional complementary disciplines $10 \%$ & & & & & \\
\hline 1. & Lied literature & & $\mathrm{x}$ & $\mathrm{x}$ & & 2 \\
\hline 2. & Stage image & & & $\mathrm{x}$ & & \\
\hline 3. & Compared performances & $\mathrm{x}$ & $\mathrm{x}$ & $\mathrm{x}$ & & 3 \\
\hline 4. & Jazz/Popular music & & & $\mathrm{x}$ & $\mathrm{x}$ & 2 \\
\hline 5. & Religious music & & & & $\mathrm{x}$ & 1 \\
\hline 6. & Musical historiography & & $\mathrm{x}$ & & $\mathrm{x}$ & 2 \\
\hline 7. & Composers and musical literature & & $\mathrm{x}$ & & $\mathrm{x}$ & 2 \\
\hline 8. & Acoustics & & & & $\mathrm{x}$ & 1 \\
\hline 9. & Theory of instruments & & $\mathrm{x}$ & & $\mathrm{x}$ & 2 \\
\hline \multirow[t]{2}{*}{10.} & Improvisation figured bass & & & & $\mathrm{x}$ & 1 \\
\hline & Operetta/musical module & & & & & \\
\hline 11. & Art of the operetta and musical performer & & & & $\mathrm{x}$ & 1 \\
\hline 12. & Workshop of choreographic creation & & & & $\mathrm{x}$ & 1 \\
\hline 13. & Operetta/musical canto & & & & $\mathrm{x}$ & 1 \\
\hline 14. & Operetta/Musical canto accompaniment & & & & $\mathrm{x}$ & 1 \\
\hline 15. & Art of musical performance & & & & $\mathrm{x}$ & 1 \\
\hline 16. & History of operetta/musical & & & & $\mathrm{x}$ & 1 \\
\hline E. & Facultative disciplines 10\% & & & & & \\
\hline \multirow[t]{2}{*}{1.} & Optional instrument & & & $\mathrm{x}$ & $\mathrm{x}$ & 2 \\
\hline & Traditional vocal music module & & & & & \\
\hline 2. & Folkloric chant & & & $\mathrm{x}$ & $\mathrm{x}$ & 2 \\
\hline 3. & Traditional ensemble & $\mathrm{x}$ & & $\mathrm{x}$ & $\mathrm{x}$ & 3 \\
\hline \multirow[t]{2}{*}{4.} & Grading and restoring traditional vocal music & & & $\mathrm{x}$ & & 1 \\
\hline & Performing module of popular/jazz music & & & & & \\
\hline
\end{tabular}




\begin{tabular}{|c|l|c|c|c|c|c|}
\hline 5. & Performing of popular/jazz music & & & $\mathrm{x}$ & & 1 \\
\hline 6. & Estrada/pop/rock ensemble & $\mathrm{x}$ & & $\mathrm{x}$ & & 2 \\
\hline 7. & Instrumental and vocal arrangement & & & $\mathrm{x}$ & & 1 \\
\hline
\end{tabular}

\section{Conclusions}

By analysing these aspects, it results that there are correlations between the curricula of the academic programs for the Musical pedagogy and Musical performance/Instruments and Canto specialisations. Through the periodical monitoring, assessment and reviewing practices of Bachelor's degree programs provided by the Romanian Agency for Quality Assurance in Higher Education, one ensures the compliance with the educational policies providing qualification according to the occupational standards for the Romanian, but also EU labour market.

The academic programs and the diplomas are elaborated according to the academic qualification demands collected on the market from admission candidates, students, graduates, employers and are issued by complying with the legal regulations in force. The qualifications were related to the academic results of the study level, formulated in terms of acquired competences, in order to determine the professional roles and then the correlation between the diplomas provided and the qualifications obtained by diploma. The European models of the artistic and musical education are considered in their entire diversity.

The constants that we notice are at the fundamental and speciality disciplines, and the large room of variables is present particularly for the complementary disciplines - compulsory and optional, as well as for the modules of facultative disciplines. The largest variation is present at the Musical performance/Instruments specialisation, generated by the instrumental categories (keyboard, string, wind, percussion) imposing particularisations. For the Musical/Canto performance specialisation, the offer has a variability coefficient by introducing modules: jazz/pop music, folkloric/traditional music, operetta/musical.

We notice that for the Musical pedagogy specialisation, the programs analysed are more homogeneous from the point of view of division on categories of disciplines and optional and facultative disciplines offer. For the Musical performance/Instruments program, the variations are higher both from the point of view of division in categories of disciplines and from the point of view of optional and facultative disciplines offer, presenting several particularities at U3 and U4. The same records are kept, but in a lower ratio and at the Musical performance/Canto program, with the same particularities at U3 and U4. The recommendations of the Romanian Agency for Quality Assurance in Higher Education are complied with, but sometimes the distribution is altered, so that some disciplines are either renamed or allocated to other categories (from fundamental to specialised or compulsory complementary), according to the regional, traditional, cultural-musical and confessional specificity. At the same time, the offer of optional and facultative disciplines is a lot more generous, provided that the students must choose, per academic year, only one discipline. At U4, we also found an offer of facultative disciplines proposed within a 
POSDRU project, that we did not quantify, having a temporary character, during the project performance. We also must add that fact that one may also cover the didactic qualification facultative program, which is not ensured by the module provided by the additional courses of the Teaching Staff Training Departments, with 30 ECTS, for the last 2 programs analysed.

As a final recommendation, we propose a higher cohesion in creating these programs, in order to support a less ratio of variables and respecting the division in categories of disciplines according to the academic standards.

\section{References}

1. http://www.aracis.ro/proceduri/ Standardele specifice pentru domeniile de licență Arte / Muzică (Specific standards for study programs in the fields of undergraduate Arts - Music) / download 09.09.2015, 23:57

2. http://www.anc.edu.ro/?page_id=40/download 07.09.2015, 21:27

3. http://www.anc.edu.ro/?page_id $=610 /$ download 07.09.2015, 21:37

4. http://www.rubinian.com/cor_4_grupa_baza.php?id=233 / download 10.09.2015, 23:10

5. http://www.rubinian.com/cor_5_ocupatia.php?id=2652/ download10.09.2015, $23: 17$

6. http://www.aracis.ro/proceduri/ Standarde specifice de evaluare academică pentru programele de studii universitare din domeniile de licență Științe administrative, Științe ale Educaţiei, Psihologie și programe a căror denumire și misiune vizează explicit pregătirea profesorilor pentru învăţământul secundar (Specific standards of academic evaluation for study programs in the fields of undergraduate Administrative Sciences, Science Education, Psychology and whose name and mission programs explicitly aimed at preparing teachers for secondary education) / download 10.10.2015, 21:20

7. http://www.arteiasi.ro/wp-content/uploads/2014/09/Muzica-Canto.pdf/ download 09.10.2015, 19:25

8. http://www.arteiasi.ro/wp-content/uploads/2014/09/Muzica-Instrumente.pdf/ download 09.10.2015, 19:30

9. http://www.arteiasi.ro/wp-content/uploads/2014/09/Muzica-Pedagogie.pdf/ download 09.10.2015, 19:35

10. http://www.unmb.ro/despre-noi/facultati-ansambluri/fim/departamentul-decanto-si-artele-spectacolului-muzical/ download 10.10.2015, 19:15

11. http://www.unmb.ro/despre-noi/facultati-ansambluri/fim/departamentul-deinstrumente-de-orchestra/ download 10.10.2015, 19:20

12. /http://www.unmb.ro/despre-noi/facultati-ansambluri/fim/departamentul-declaviaturi-si-muzica-de-camera/ download 10.10.2015, 19:25

13. http://www.unmb.ro/despre-noi/facultati-ansambluri/fcmpm/departamentulde-muzicologie-si-stiintele-educatiei-muzicale/download 10.10.2015, 19:30 14. http://www.amgd.ro/upload/files/ExtrasPlanInvLicentaIM2015-2016.pdf/ download 11.10.2015, 19:20 
15. http://www.amgd.ro/upload/files/ExtrasPlanInvLicentaCANTO20152016.pdf/ download 11.10.2015, 19:25

16. http://www.amgd.ro/upload/files/ExtrasPlanInvLicentaPeda2015-2016.pdf

17. http://fmt.uvt.ro/wp-content/uploads/2014/07/PI_IMI.pdf/_ download

12.10.2015, 19:20

18. http://fmt.uvt.ro/wp-content/uploads/2014/07/PI_PM.pdf/_ download 12.10.2015, 19:25

19. http://muzica.dev.uvt.ro/wp-content/uploads/2014/07/PI_IMC.pdf/download 12.10.2015, 19:30

20. http://www.emanuel.ro/wp-content/uploads/2016/03/Ghidul-Admiterii-2016 -2017. pdf/ download 12.10.2015, 19:35

21. http://www.valahia.ro/ro/facultati/29-facultatea-de-stiinte-si-arte/ download $13.10 .2015,19: 25$

22. http://eacea.ec.europa.eu/education/eurydice/documents/thematic_reports/13 8RO.pdf / download 14.10.2015, 19:30

23. http://eacea.ec.europa.eu/education/eurydice/documents/thematic_reports/12 2RO.pdf / download 14.10.2015, 19:45

24. Bologna Process Stocktaking Report Report from working groups appointed by the Bologna Follow-up Group to the Ministerial Conference in Leuven/Louvain-la-Neuve, 2009

25. HG 1352/2010 privind aprobarea structurii Clasificării ocupaţiilor din România - nivel grupa de bază, conform Clasificării internaționale standard a ocupatiilor - ISCO 08 (regarding the approval of the Classification of Occupations in Romania - based group level, according to the International Standard Classification occupations - ISCO 08) 